\title{
Identification of texture MRI brain abnormalities on first-episode psychosis and clinical high-risk patients using explainable artificial intelligence
}

\author{
Alexandra Korda ( $\square$ alexandra.korda@uksh.de) \\ UKSH Luebeck
}

Christina Andreou

UKSH Luebeck https://orcid.org/0000-0002-6656-9043

Helena Victoria Rogg

UKSH Luebeck

Mihai Avram

UKSH Luebeck

Anne Ruef

LMU

Christos Davatzikos

University of Pennsylvania https://orcid.org/0000-0002-1025-8561

Nikolaos Koutsouleris

LMU https://orcid.org/0000-0001-6825-6262

\section{Stefan Borgwardt}

UKSH Luebeck

\section{Article}

Keywords: First-episode psychosis, clinical high-risk, brain sMRI, biomarkers, texture, MRI, deep learning, layer-wise relevance propagation

Posted Date: March 3rd, 2022

DOI: https://doi.org/10.21203/rs.3.rs-1380386/v1

License: (c) (1) This work is licensed under a Creative Commons Attribution 4.0 International License. Read Full License

Version of Record: A version of this preprint was published at Translational Psychiatry on November 16th, 2022. See the published version at https://doi.org/10.1038/s41398-022-02242-z. 


\section{Abstract}

Structural MRI studies in first-episode psychosis and the clinical high-risk state have consistently shown volumetric abnormalities. Aim of the present study was to introduce radiomics texture features in identification of psychosis. Radiomics texture features describe the interrelationship between voxel intensities across multiple spatial scales capturing the hidden information of underlying disease dynamics in addition to volumetric changes. Structural MR images were acquired from 77 first-episode psychosis (FEP) patients, 58 clinical high-risk patients with no later transition to psychosis (CHR_NT), 15 clinical high-risk patients with later transition (CHR_T) and 44 healthy controls (HC). Radiomics texture features were extracted from non-segmented images, and two classification schemas were performed for the identification of FEP vs. HC and FEP vs. CHR_NT. The group of CHR_T was used as external validation in both schemas. The classification of a subject's clinical status was predicted by importing separately a) the difference of entropy feature map and b) the contrast feature map, resulting in classification accuracy above $72 \%$ in both analyses. The proposed framework enhances the classification decision for FEP, CHR_NT and HC subjects, verifies diagnosis-relevant features and may potentially contribute to identification of structural biomarkers for psychosis, beyond and above volumetric brain changes.

\section{Introduction}

Structural brain abnormalities have been associated with schizophrenia and volume deficits progress across the trajectory of the illness ${ }^{1-3}$. These changes have been observed in early stages of psychosis as in first-episode psychosis patients (FEP) ${ }^{4-6}$, have a wide distribution, affecting frontal, temporal, parietal cortical regions, but also subcortical, cerebellar and callosal regions ${ }^{7-9}$, and have a progressive course ${ }^{10}$. Several studies have identified similar volumetric disturbances in populations at high risk for psychosis ${ }^{11,12}$.

Psychosis risk definitions based on clinical criteria (clinical high risk, CHR) have low specificity for prediction of a future transition to overt psychosis, with only about a quarter of patients developing a psychotic episode 3 years after diagnosis ${ }^{13,14}$. Consequently, there has been major interest in brain structural changes as biomarkers useful for predicting the future emergence of psychosis in CHR with higher accuracy than clinical criteria alone. Several studies and meta-analyses that investigated grey and white matter alterations in CHR have confirmed their potential as predictive indices, identifying a number of differences between $\mathrm{CHR}$ with later transition compared to those without with respect to total grey matter volume ${ }^{15,16}$, specific regions such as the anterior cingulate ${ }^{17,18}$, frontal cortex ${ }^{19,20}$, temporal cortex ${ }^{21}$, parietal cortex ${ }^{22}$, cerebellum ${ }^{23}$, and insular cortex ${ }^{24}$, but also white matter volume and structure ${ }^{25-27}$.

The majority of the above studies have focused on regional volume or intensity measures and have not fully exploited the rich information contained in brain MRI, e.g. subtle differences between brain tissues or 
in the microstructure of the biological tissue ${ }^{28}$, complex interrelations between different regions or grey, white matter and $\mathrm{CSF}^{29}$. Radiomics texture features are able to quantify the hidden patterns between voxel intensities and the spatial distribution of these patterns across brain regions. Radiomics texture features with their potential as image-based biomarkers have been widely used across several studies, like for cancer identification ${ }^{30}$, Alzheimer's ${ }^{31}$ and Parkinson's disease ${ }^{32}$ as neurodegenerative diseases, major depression ${ }^{33}$ and schizophrenia ${ }^{34,35}$. In the field of schizophrenia research, texture features such as homogeneity and entropy have been shown to differentiate patients from healthy controls $(\mathrm{HC})^{36}$. The main advantage of applying radiomics texture features is their potential to capture microscopic alterations in tissue characteristics of the brain ${ }^{37}$, even though authors have stressed the significance of repeatability and reproducibility in applying texture radiomics features ${ }^{38,39}$.

In the current study, we applied radiomics texture features on FEP and CHR brain images for the first time. We examined potential differences between FEP, CHR with later transition to psychosis (CHR_T), CHR with no transition to psychosis (CHR_NT), and HC, by employing six texture feature maps extracted from nonsegmented MR images and feeding into a deep neural network binary classification schema. For classification, we employed an innovative approach that addresses a frequent concern about artificial intelligence methods, i.e. the explainability of results. Our proposed algorithm integrates the complexity of the deep neural networks with the explainability of these networks as introduced by Bach et al. ${ }^{40}$. The algorithm, which is applied on non-segmented brain MRI for the investigation of the inter-relation between white matter (WM), GM and cerebrospinal fluid (CSF) has been previously reported for the identification

of schizophrenia and major depression in Korda et al. ${ }^{41}$. Based on previous findings ${ }^{42,43}$, we hypothesize that radiomics texture feature models (a) discriminate FEP patients from healthy controls and CHR patients without a later transition to psychosis, and (b) predict later transition in CHR patients based on the FEP pattern.

\section{Methods}

\subsection{Study participants}

The current analyses are based on data from the early detection of psychosis project (FePsy) at the Department of Psychiatry, University of Basel, Switzerland ${ }^{44}$. FEP, CHR patients with (CHR-T) and without later transition to psychosis (CHR-NT) and healthy controls (HC) were recruited from November 2008 to April 2014. The Basel Screening Instrument for Psychosis (BSIP) was used for assessment of CHR and FEP status. The BSIP is a 46-item instrument based on variables that have been shown to be risk factors for early symptoms of psychosis such as DSM-III-R - "prodromal symptoms," social decline, drug abuse, previous psychiatric disorders, or genetic liability for psychosis ${ }^{45}$. CHR status was defined based on the presence of either attenuated psychotic symptoms, brief limited intermittent psychotic symptoms, or having a first- or second-degree relative with a psychotic disorder plus at least 2 additional risk factors for psychosis. FEP status was defined according to criteria for transition to psychosis by Yung et al. ${ }^{46}$. 
Structural MRI scans of 194 subjects were used for analysis: 77 FEP, 58 CHR_NT, 15 CHR_T and 44 HC. Subjects were scanned using a SIEMENS (Erlangen, Germany) MAGNETOM VISION 1.5T scanner at the University Hospital, Basel. A three-dimensional volumetric spoiled gradient recalled echo sequence generated 176 contiguous, 1 - mm-thick sagittal slices. Imaging parameters were time-to-echo, $4 \mathrm{~ms}$; timeto-repetition, $9.7 \mathrm{~ms}$; flip angle, $12 \circ$; matrix size, $200 \times 256$; field of view, $25.6 \times 25.6 \mathrm{~cm}$ matrix; voxel dimensions, $1.28 \times 1 \times 1 \mathrm{~mm}$. Inclusion and exclusion criteria are described in detail in ${ }^{4}$.

\subsection{MRI data acquisition and data preprocessing}

After inspection for artifacts and gross abnormalities, MRI scans were segmented into GM, WM, and CSF tissue maps in native space by means of the Cat12toolbox (http://dbm.neuro.uni-jena.de), an extension of the SPM12 software package (Wellcome Department of Cognitive Neurology, London, England). All scans were reviewed by a neuroradiologist to rule out clinically significant abnormalities. The process was automated and has been described in Koutsouleris et al. ${ }^{47}$ and Koutsouleris et al. ${ }^{48}$. Computation time of the preprocess was less than 30 minutes per subject.

It is critical to keep potential variances in image pose variance entering texture feature maps calculations to a minimum as these have been noted to affect texture estimates. In detail, the CAT12 toolbox extends the unified segmentation model consisting of MRI field intensity inhomogeneity correction, spatial normalization and tissue segmentation in several preprocessing steps to further improve the quality of data preprocessing. Initially, the Optimized Blockwise Nonlocal-Means filter proposed by Coupe at al. 49 was applied to the MRI scans using the Rician noise adaption introduced in Wiest-Daesslé et al. ${ }^{50}$ to increase the signal-to-noise ratio in the data. Subsequently, an adaptive maximum a posteriori segmentation approach ${ }^{51}$ extended by partial volume estimation ${ }^{52}$ was employed to separate the MRI scans into GM, WM, CSF tissue. The segmentation step was finished by applying a spatial constraint to the segmented tissue probability maps based on a hidden Markov Random Field model ${ }^{53}$ that removed isolated voxels which were unlikely to be a member of a certain tissue class and closed gaps in clusters of connected voxels of a certain class, resulting in a higher signal-to-noise ratio of the final tissue probability maps. The original voxels are projected into their new location in the warped images preserving the volume of a particular tissue within a voxel (i.e., produced by affine transformation (global scaling) and non-linear warping (local volume change), but this has the effect of introducing aliasing artifacts ${ }^{54}$. This latter effect was eliminated by applying discretization of the intensities in the mwp0* images, which is similar to applying a smoothing filter on the data distribution ${ }^{55}$.

We use histogram equalization to adjust the contrast of a greyscale image. The original image has low contrast, with most pixel values in the middle of the intensity range. The histeq function in Matlab produces an output image with pixel values evenly distributed throughout the range (Fig. 1 in Supplement). In Fig. 1, the difference in the intensity between the initial and the transformed image is presented. The texture feature maps were extracted from the transformed mwp0* image (see Fig. 2 for workflow). 


\subsection{Texture Feature Map}

\subsubsection{Adjust Intensity Values Using Histogram Equalization}

We extracted textural parameters from non-segmented images using grey-level co-occurrence matrix (GLCM) ( $N=1,164$ feature maps in total). We extracted the texture features Entropy, Sum of Entropy, Difference of Entropy, Energy, Contrast, Homogeneity ${ }^{56}$. The definitions of these features were as follows: GLCM-contrast reflects local variations in the GLCM; GLCM-energy reflects uniformity of greylevel voxel pairs; GLCM-entropy reflects randomness of grey-level voxel pairs; and GLCM-homogeneity reflects homogeneity of grey-level voxel pairs ${ }^{57,58}$; finally GLCM-sum of entropy and difference of entropy reflect second order statistics of differentiation of grey level distribution GLCM. We used voxel-by-voxel sliding 3D cube of $7 x 7 x 7$ dimension as presented in a previous paper ${ }^{33}$. The GLCM matrix was normalized by dividing the values with the total sum of the values in the matrix. The normalization was performed for each GLCM extracted in each 3D cube independently.

All the feature maps calculated from the $2 \mathrm{~d}$ GLCM were basically a function of the probability of each GLCM entry and the difference of the grey levels, $g_{1}$ and $g_{2}{ }^{59}$. We calculated feature maps only for cubes including non-zero values, as presented in Appendix A. The registered texture feature maps on the MNI space were fed into a 10x10 nested cross-validation deep learning scheme for group classification (see Fig. 2 in Supplement).

We focus the description of results on the difference of entropy feature, which measures the randomness of intensity distribution in a second level, inherits the characteristics of entropy, and typically is less sensitive to outliers in a region ${ }^{28}$. The contrast gives to elements with similar grey level values a low weight but elements with dissimilar grey levels a high weight, indicating large differences between neighboring voxels ${ }^{60}$.

\subsection{Deep learning}

The deep learning technique utilized the registered radiomics texture feature maps as input. We implemented two cycles of 10-times repeated nested cross-validation with 10 folds in the inner cycle and 10 folds in the outer cycle, resulting in 10'000 models. Feature selection (two-sample t-test) in the inner cycle, was cross-validated by selecting a number of features appropriate to the dimension of the database, namely, the top 200 ranked features that best discriminated the 2 classes in each classification schema (see section 3.2) ${ }^{61}$. The classifier implemented was a neural network-based classifier implemented in MATLAB (MathWorks Inc., Natick, Massachusetts, USA). The network used the hyperbolic tangent sigmoid transfer function and was batch-trained using the Levenberg-Marquardt training algorithm ${ }^{62}$. L2-regularization was applied to access possible types of uncertainty. We selected parameters after experimentation; 5 hidden layers (tested 2 to 5 ), each hidden layer consists of 2 nodes (tested 2 to 20 ) and 1,000 epochs. The average accuracy, sensitivity and specificity was calculated across all hold-out datasets of the 10x10 nested cross-validation, repeated 10 times. 


\subsection{Visualization and evaluation of heatmaps}

In order to perform localization, we calculated the relevance of the voxels in each class using the LRP algorithm for multilayer neural networks, as described in Bach et al. ${ }^{40}$. The explanation given by LRP would be a map showing which voxels of the original texture feature map contribute to the diagnosis and to what extent.

For the specific deep learning schema with 5 hidden layers and size 2, the calculation of the LRP algorithm is presented in Appendix B. The output of the LRP algorithm is a heatmap for each subject representing change in brain structures for FEP, CHR_NT and CHR_T patients. The final images were smoothed with a Gaussian filter of $10 \mathrm{~mm}$ and visualized using the MRIcron toolbox (https://people.cas.sc.edu/rorden/mricron/install.html). Similarities of the heatmaps for subjects in the same group were identified by clustering the heatmaps. Evaluation of the clustered heatmaps gives an estimation to the importance of each brain region to the diagnostic group membership (Figs. 3-5). Visualizations of the classification results on the holdout dataset are presented in Figs. 3-5. The regions of interest were extracted using the AAL-VOls atlas (https://neurovault.org/images/14257/). Additionally, we investigated cortical biomarkers for psychosis in GM, WM and CSF. We used the JHU WM tractography atlas ${ }^{63}$ to identify the WM tractography identified by the LRP.

\subsection{Clustering of Subjects}

Our intention was display the heatmap of each correctly classified subject in the hold-out testing set produced by the LRP algorithm in a grouped fashion ${ }^{64}$. The affinity propagation (AP) algorithm ${ }^{65}$ was selected to cluster the subject's positive relevance, which uses the concept of message passing between the samples. The main advantage of the AP algorithm is that the number of clusters is not predefined. The input in the clustering algorithm is a matrix $M \times N$, where $N$ is the number of subjects and $M$ is the relevance of each voxel. The output of the AP algorithm is a scalar for every subject that expresses in which cluster the subject belongs to. The average of the heatmaps from the subjects belonging to each cluster are represented.

For each classification process, the relevancies were classified using the AP algorithm. The clusters are presented in Fig. 3-5 for each texture feature map under the two-classification schema. The red color corresponds to the most intense cluster, the second one is in blue color and the green is the third one, where it exists (see Fig. 3-5).

\section{Results}

\subsection{Sociodemographic characteristics}

There were no significant differences between FEP and $\mathrm{HC}$ with respect to age and alcohol consumption. For the comparison between FEP and CHR, there were no significant differences with respect to age, years 
of education, smoking, alcohol consumption and gender. There were significant differences between FEP and CHR with respect to BPRS total, BPRS Negative and Positives Symptoms and SANS total (see Table 1).

\section{Table 1}

Group comparison using 1-way ANOVA for continuous and $\chi^{2}$ test for categorical data.

\section{One-Way ANOVA (Welch's)}

\begin{tabular}{lll} 
FEP vs HC & F & P \\
\hline Age & 1.63 & 0.204 \\
\hline Education (Years) & 19.26 & $<.001$ \\
\hline Smoking (Cigarettes per Day) & 28.18 & $<.001$ \\
\hline FEP vs CHR & & \\
\hline Age & 3.118 & 0.056 \\
\hline Education (Years) & 0.165 & 0.849 \\
\hline Global Assessment of Functioning (GAF) & 46.875 & $<.001$ \\
\hline BPRS_Positive_Symptoms & 19.498 & $<.001$ \\
\hline BPRS_Negative_Symptoms & 22.494 & $<.001$ \\
\hline BPRS_total & 462.930 & $<.001$ \\
\hline SANS_total & 128.957 & $<.001$ \\
\hline Smoking (Cigarettes per Day) & 2.418 & 0.102 \\
\hline$\chi^{2}$ Tests & &
\end{tabular}

\section{FEP vs HC}

Sex

13.6

$<.001$

Alcohol

9.60

0.008

FEP vs CHR

Sex

0.431

0.511

Alcohol

2.80

0.246

Note: BPRS, Brief Psychiatric Rating Scale; GAF, Global Assessment of Functioning; SANS, Scale for the Assessment of Negative Symptoms

\subsection{Classification results and visualization}


Two classifiers were developed in this study: a) FEP vs. HC and b) FEP vs. CHR_NT. The average accuracy, sensitivity and specificity in the outer cycle were calculated for 10 folds in 10 repetitions. We tested the texture feature maps one-by-one in the deep learning classifier (see Tables 2-3). The specificity for FEP compared to HC (classifier a) was above 75\% across features (Tables 2 and 3 ). The sensitivity in the classification schema $b$ was above $70 \%$ across features, indicating adequately correct classification of FEP against CHR_NT.

Table 2

presents the accuracy, sensitivity and specificity of the hold-out testing set in schema a.

\begin{tabular}{|llll|}
\hline Registered texture feature map & Accuracy (\%) & Sensitivity (\%) & Specificity (\%) \\
\hline Entropy & 66.55 & 56.93 & 75.08 \\
\hline Sum of entropy & 72.02 & 68.22 & $\mathbf{8 1 . 9 2}$ \\
\hline Difference of entropy & 74.56 & 69.58 & 88.02 \\
\hline Contrast & 75.24 & 76.48 & 83.63 \\
\hline Homogeneity & 70.43 & 63.87 & 77.23 \\
Energy & 70.29 & 60.71 & $\mathbf{8 0 . 0 8}$ \\
\hline
\end{tabular}

Table 3

presents accuracy, sensitivity and specificity the hold-out testing set in schema b.

\begin{tabular}{|llll|}
\hline Registered texture feature map & Accuracy (\%) & Sensitivity (\%) & Specificity (\%) \\
\hline Entropy & 64.52 & 70.01 & 62.90 \\
\hline Sum of entropy & 68.48 & 71.49 & 73.20 \\
\hline Difference of entropy & 72.01 & 79.29 & 71.00 \\
\hline Contrast & 73.21 & 78.95 & 71.92 \\
\hline Homogeneity & 70.52 & 78.59 & 68.06 \\
\hline Energy & 70.24 & 75.59 & 72.30 \\
\hline
\end{tabular}

In external validation using CHR-T, the most discriminative features were the difference of entropy and contrast resulting in an accuracy above $72 \%$; these features are presented in further detail for the interpretation of the results below; for completeness, analysis of the remaining texture features is presented in Supplement. Applying the classification schema a, the feature difference of entropy classified 10 out of 15 CHR_T as FEP, while with the classification schema $b$, the feature difference of entropy classified 11 out of 15 CHR_T as FEP patients.

\subsection{Clustering of subjects}


Across texture feature maps, regions with the highest contribution to the difference between FEP and HC (i.e. those with highest positive relevance PR) were in cerebellum and frontal gyrus (contrast), lenticular fasciclus (difference of entropy), see Fig. 3. In addition, regions most contributing to the difference between FEP and CHR_NT patients were in parahippocampal, amygdala, precuneus, caudate, putamen, thalamus, hippocampus, insula, cerebellum, vermis, pallidum lingual and motor area (difference of entropy and contrast), see Fig. 4. By explaining the classification decision using the LRP algorithm for the four models performed using the difference of entropy map and contrast map in classification schemas $a$ and $b$, the cerebellum was observed to be the key region for psychosis. Furthermore, key regions for psychosis were observed that are in line with previous studies, e.g., amygdala, caudate, insula, and hippocampus.

Regions contributing most to the CHR_T classification as FEP using the difference of entropy texture feature map (Fig. 5) were in parahippocampal areas, amygdala, precuneus, caudate, putamen, thalamus, hippocampus, insula, cerebellum, vermis, pallidum, lingual and motor area. Furthermore, for the classification schema a, two clusters of regions could be identified using the difference of entropy map. Cluster 1 included brain regions that were not part of the cluster 2 (Table 4), indicating a distinct neurobiological profile for subjects grouped in cluster 1 .

Table 4

presents the regions revealed in one cluster in schema a grouped fashion visualization.

\begin{tabular}{|ll|}
\hline $\begin{array}{ll}\text { FEP vs HC } \\
\text { Difference of entropy map }\end{array}$ \\
\hline Anterior corona radiata left & Cluster 1 \\
\hline Insular left & Cluster 1 \\
\hline Lateral fronto-orbital gyrus left & Cluster 1 \\
\hline Middle fronto-orbital gyrus left & Cluster 1 \\
\hline Gyrus rectus left & Cluster 1 \\
\hline Appendices & \\
\hline Appendix A & \\
\hline
\end{tabular}

We identified no consistent correlations of the PR with the volume of the regions indicated by the LRP when analyzed in the hold-out datasets that concluded to the highest accuracy. The PR was negatively correlated with the volume of the regions indicated by the LRP for FEP subjects in classification schema a) (Fig. 6 in Supplement), such as optic tract left, corticospinal tract right, hypothalamus right (difference of entropy). Volumetric changes of regions such as calcarine left, fusiform right and cuneus left (contrast) were correlated positively with PR. In classification schema b) (Fig. 7 in Supplement), the PR for the FEP subjects was positively correlated with temporal and vermis (difference of entropy), frontal 
and precentral left (contrast) and uncorrelated with insula left, parahippocampal left, caudate left, calcarine left, Heschl left, cuneus left, putamen and thalamus left, lingual right (difference of entropy), frontal, temporal and parietal cortex and supplementary motor area left (contrast). PR was uncorrelated with volumetric changes across CHR_NT that classified correctly against FEP such as thalamus, insula, putamen, hippocampus and parahippocampal, caudate and amygdala (difference of entropy). CHR patients who made a transition to psychosis and were classified as FEP with high accuracy using the difference of entropy map in classification schema $b$ ), presented uncorrelated volumetric changes with PR in many regions, i.e., thalamus, amygdala left, putamen, insula and others shown in Fig. 8 in Supplement. These regions were the dominant regions for the prediction of psychosis based on baseline MRI.

For classification schema a) (Fig. 3) LRP revealed PR in regions such as anterior corona radiata and genu of CC (contrast), posterior limb of internal capsule right, superior corona radiata right, superior fronto occipital fasciculus (difference of entropy). Regions such as cerebral peduncle right, posterior limb of internal capsule right and external capsule right (difference of entropy), body of CC, superior corona radiata right and superior longitudinal fasciculus right (contrast) contribute more to the identification of the FEP subjects in classification schema b) (Fig. 4) and the CHR_T patients that classified as FEP subjects (Fig. 5). Across classification schemas, the WM volumetric changes did not impact the values of the PR indicating that texture features capture the dynamic inter-relation between GM, WM and CSF with predictive power for psychosis. Changes in the CSF captured by the difference of entropy for the FEP against CHR_NT (Fig. 4) and CHR_T classified as FEP (Fig. 5).

\section{Discussion}

In this study, we used radiomic texture feature maps and the explainable Al method suggested by Bach et al. ${ }^{40}$ to train and explain a classifier for psychosis. The model showed high accuracy in classifying CHR patients with a later transition as FEP rather than healthy subjects, thus indicating a potential use for predictive purposes. Importantly, texture features were not correlated with volumetric changes in a consistent manner, suggesting that this measure can reveal hidden neuro-biological patterns expanding beyond volumetric changes of single regions to include the interrelations and borders between GM, WM and CSF.

Our findings are in line with a previous study applying texture analysis on MR data in patients with schizophrenia, which reported altered entropy in the hippocampus and the amygdala ${ }^{36}$. The regions contributing mostly to the decision of our classifier included key regions implicated in psychotic disorders in studies that have assessed grey and white matter changes in patients.

- Grey matter. Brain alterations with a decrease of intracranial and total brain volume have been reported in patients with chronic schizophrenia ${ }^{66}$, particularly affecting cortical gray matter (and here predominantly in the prefrontal cortex (PFC) ${ }^{67}$. Some of these changes such as volume decrease in the thalamus have been also observed in FEP ${ }^{68}$, which emphasizes the presence of 
brain alterations already in early stages of the disease. These alterations might be pertinent to the transition to psychosis in CHR patients: For example, CHR-T showed more deterioration over time in frontal and temporal regions than CHR_NT in early studies ${ }^{69,70}$. In other regions such as the insular or cingulate cortex, grey matter loss in CHR-T has also been reported to exceed that of CHR_NT ${ }^{71}$. The structural alterations reported in previous studies are in line with the identified regions in the current analysis, such as temporal regions ${ }^{72}$; frontal cortex ${ }^{73-75}$; thalamus ${ }^{68}$; insula ${ }^{76}$;

hippocampus and caudate nucleus ${ }^{77,78}$; pallidum, putamen ${ }^{79}$; parahippocampus ${ }^{79,80}$; and lingual cortex ${ }^{81}$.

- White matter. There is accumulating evidence of compromised white matter function leading to abnormalities in synchronization and connectivity in patients with schizophrenia ${ }^{82}$, the most widely used measure being fractional anisotropy (FA) assessed with diffusion tensor imaging (DTI). Neural changes, especially changes in the white matter connectivity could be observed throughout the different stages and progress of psychosis ${ }^{83}$. There are consistent findings of decreased FA, particularly in the inferior-fronto-occipital fasciculus (IFOF) ${ }^{84,85}$. Other studies have reported reduced white matter volume ${ }^{86}$ and decreased FA in superior longitudinal fasciculus (SLF) ${ }^{87}$ as well as in inferior fronto-orbital fasciculus (IFOF) ${ }^{88-90}$, in CHR compared to FEP.

Multiple texture maps reveal significant contribution in diagnostic group membership for FA and IFOF, for both FEP and CHR_NT who classified as FEP. The above findings are in line with our results regarding the superior longitudinal fasciculus and inferior fronto-orbital fasciculus using contrast maps, and the thalamus, insula, hippocampus, pallidus, putamen and parahippocampus using the difference of entropy maps. To exclude the possibility that our findings simply reflect volume reductions in these regions, we investigated whether regions contributing most to the diagnostic group membership (i.e. regions with PR values) were correlated with changes in brain volume in individuals with FEP and CHR (see Fig. 6-8 in Supplement. We found no indication of correlation of the relevance of the regions in the diagnostic group membership with volumetric changes in specific areas, i.e. amygdala, putamen, thalamus, hippocampus, insula, pallidus, rectus, parahippocampus, lingula and Heschl.

The localization of alterations appeared to differ between patients in different illness stages using the difference of entropy map: whereas most prominent alterations in FEP involved the cingulate gyrus and subcortical regions such as the nigrostriatal circuit, amygdala and hypothalamus (Fig. 3a), CHR with later transition to psychosis demonstrated alterations mainly in cortical regions, the thalamus, and cerebellum (Fig. 4a). Both grey matter and white matter loss in specific brain regions were more prominent in $\mathrm{CHR}$ with transition to psychosis (CHR_T) compared to CHR who did make the transition. These brain alterations considered of high clinical relevance, resulting in - for example - more severe positive and negative symptoms and worse social functioning ${ }^{87,91}$.

Regarding implementation, the variability in standardization of MRI intensities influences the extraction of the texture features. In this study, the radiomic texture feature maps were extracted from the registered masked T1-weighted image in MNI space (as opposed to the original space as in Korda et al. ${ }^{41}$. 
Parameter selections such as the radius of the cubes and the structure of the neural network should be further examined. Regarding the LRP explanations, difference of entropy was the dominant texture feature map for psychosis. However, we recommend that further studies use multiple texture features, as each one expresses a different dynamic of brain heterogeneity. In summary, different methodological options need to be further explored in order to get a better understanding of the neurobiological changes in psychosis and their course from the CHR to the FEP stage, in order to make findings relevant for targeted interventions and individual treatment options. To investigate whether different neuroimaging modalities can be combined and used for increased accurate prediction, further work is required and should be addressed in future studies.

Another unresolved question concerns the effects of antipsychotic medication ${ }^{3}$. Several studies have shown that antipsychotic medication has an impact on alterations in WM disturbances between treated patients, drug-naïve patients and healthy controls ${ }^{92,93}$, while smaller brain tissue volumes and larger cerebrospinal fluid volumes can be observed in long-term treatment with antipsychotics ${ }^{94}$. It is still unclear whether brain abnormalities are already present at early stages and probably predicting the clinical onset of schizophrenia, or if these changes occur during the course of illness or are caused by pharmacological treatment ${ }^{95}$. It is crucial that the nature, time occurrence and further course of such brain changes as well as the impact of antipsychotic treatment is further investigated.

\section{Conclusions}

Conventional imaging parameters are inadequate for quantification of the spatial distribution of microscopic tissue heterogeneity. A promising alternative to improve the diagnosis of psychosis on the basis of neurobiology is the application of radiomics texture features. We investigated the relation between neurobiological markers and LRP explanations. We observed that texture feature maps can be a useful representation for characterizing dissimilarities in brain structure in a complementary manner to volumetric analysis. Further studies in large cohorts are warranted to establish the key regions and key texture features that characterize psychotic disorders, in order to improve our understanding of the neurobiological changes that occur before the onset of psychosis and promote research on prevention and treatment methods for CHR patients.

\section{Declarations}

\section{Acknowledgments}

Computational support and infrastructure provided by the OMICS at the University of Luebeck (Germany). Conflict of interest The authors declare no conflict of interest.

\section{References}


1. Borgwardt, S. J. et al. Regional Gray Matter Volume Abnormalities in the At Risk Mental State. Biological Psychiatry 61, 1148-1156, doi:https://doi.org/10.1016/j.biopsych.2006.08.009 (2007).

2. Fusar-Poli, P. et al. Transdiagnostic psychiatry: a systematic review. World Psychiatry 18, 192-207, doi:https://doi.org/10.1002/wps.20631 (2019).

3. Chopra, S. et al. Differentiating the effect of antipsychotic medication and illness on brain volume reductions in first-episode psychosis: A Longitudinal, Randomised, Triple-blind, Placebo-controlled MRI Study. Neuropsychopharmacology 46, 1494-1501, doi:10.1038/s41386-021-00980-0 (2021).

4. Borgwardt, S. et al. Distinguishing prodromal from first-episode psychosis using neuroanatomical single-subject pattern recognition. Schizophrenia bulletin 39, 1105-1114, doi:10.1093/schbul/sbs095 (2013).

5. Fusar-Poli, P. et al. Cognitive Functioning in Prodromal Psychosis: A Meta-analysis. Archives of General Psychiatry 69, 562-571, doi:10.1001/archgenpsychiatry.2011.1592 (2012).

6. Ruef, A. et al. Magnetic resonance imaging correlates of first-episode psychosis in young adult male patients: combined analysis of grey and white matter. J Psychiatry Neurosci 37, 305-312, doi:10.1503/jpn.110057 (2012).

7. Radua, J. et al. Multimodal meta-analysis of structural and functional brain changes in first episode psychosis and the effects of antipsychotic medication. Neuroscience \& Biobehavioral Reviews 36, 2325-2333, doi:https://doi.org/10.1016/j.neubiorev.2012.07.012 (2012).

8. Gallardo-Ruiz, R., Crespo-Facorro, B., Setién-Suero, E. \& Tordesillas-Gutierrez, D. Long-Term Grey Matter Changes in First Episode Psychosis: A Systematic Review. Psychiatry investigation 16, 336345, doi:10.30773/pi.2019.02.10.1 (2019).

9. Arnone, D., Mclntosh, A. M., Tan, G. M. Y. \& Ebmeier, K. P. Meta-analysis of magnetic resonance imaging studies of the corpus callosum in schizophrenia. Schizophrenia Research 101, 124-132, doi:https://doi.org/10.1016/j.schres.2008.01.005 (2008).

10. Vita, A., De Peri, L., Deste, G. \& Sacchetti, E. Progressive loss of cortical gray matter in schizophrenia: a meta-analysis and meta-regression of longitudinal MRI studies. Translational Psychiatry 2, e190e190, doi:10.1038/tp.2012.116 (2012).

11. Del Re, E. C. et al. Baseline Cortical Thickness Reductions in Clinical High Risk for Psychosis: Brain Regions Associated with Conversion to Psychosis Versus Non-Conversion as Assessed at One-Year Follow-Up in the Shanghai-At-Risk-for-Psychosis (SHARP) Study. Schizophrenia Bulletin 47, 562574, doi:10.1093/schbul/sbaa127 (2020).

12. Ellis, J. K., Walker, E. F. \& Goldsmith, D. R. Selective Review of Neuroimaging Findings in Youth at Clinical High Risk for Psychosis: On the Path to Biomarkers for Conversion. Frontiers in Psychiatry 11, doi:10.3389/fpsyt.2020.567534 (2020).

13. Suvisaari, J. et al. Is It Possible to Predict the Future in First-Episode Psychosis? Frontiers in Psychiatry 9, doi:10.3389/fpsyt.2018.00580 (2018).

14. Beck, K. et al. Clinical and functional long-term outcome of patients at clinical high risk (CHR) for psychosis without transition to psychosis: A systematic review. Schizophrenia Research 210, 39-47, 
doi:https://doi.org/10.1016/j.schres.2018.12.047 (2019).

15. Smieskova, R. et al. Neuroimaging predictors of transition to psychosis-A systematic review and meta-analysis. Neuroscience \& Biobehavioral Reviews 34, 1207-1222, doi:https://doi.org/10.1016/j.neubiorev.2010.01.016 (2010).

16. Vissink, C. E. et al. Structural brain volumes of individuals at clinical high risk for psychosis: a metaanalysis. Biological Psychiatry Global Open Science, doi:https://doi.org/10.1016/j.bpsgos.2021.09.002 (2021).

17. Meijer, J. H. et al. Semantic fluency deficits and reduced grey matter before transition to psychosis: A voxelwise correlational analysis. Psychiatry Research: Neuroimaging 194, 1-6, doi:https://doi.org/10.1016/j.pscychresns.2011.01.004 (2011).

18. Yücel, M. et al. Morphology of the anterior cingulate cortex in young men at ultra-high risk of developing a psychotic illness. British Journal of Psychiatry 182, 518-524, doi:10.1192/bjp.182.6.518 (2003).

19. Allen, P. et al. Transition to Psychosis Associated With Prefrontal and Subcortical Dysfunction in Ultra High-Risk Individuals. Schizophrenia Bulletin 38, 1268-1276, doi:10.1093/schbul/sbr194 (2012).

20. Merritt, K., Luque Laguna, P., Irfan, A. \& David, A. S. Longitudinal Structural MRI Findings in Individuals at Genetic and Clinical High Risk for Psychosis: A Systematic Review. Frontiers in Psychiatry 12, 49 (2021).

21. Allen, P. et al. Emerging Temporal Lobe Dysfunction in People at Clinical High Risk for Psychosis. Frontiers in Psychiatry 10, 298 (2019).

22. Zikidi, K. et al. Grey-matter abnormalities in clinical high-risk participants for psychosis. Schizophrenia Research 226, 120-128, doi:https://doi.org/10.1016/j.schres.2019.08.034 (2020).

23. Zarogianni, E., Storkey, A. J., Johnstone, E. C., Owens, D. G. C. \& Lawrie, S. M. Improved individualized prediction of schizophrenia in subjects at familial high risk, based on neuroanatomical data, schizotypal and neurocognitive features. Schizophrenia Research 181, 6-12, doi:https://doi.org/10.1016/j.schres.2016.08.027 (2017).

24. Takahashi, T. et al. Progressive Gray Matter Reduction of the Superior Temporal Gyrus During Transition to Psychosis. Archives of General Psychiatry 66, 366-376, doi:10.1001/archgenpsychiatry.2009.12 (2009).

25. Wible, C. G. et al. Prefrontal cortex, negative symptoms, and schizophrenia: an MRI study. Psychiatry research 108, 65-78, doi:10.1016/s0925-4927(01)00109-3 (2001).

26. Chung, Y. et al. Use of Machine Learning to Determine Deviance in Neuroanatomical Maturity Associated With Future Psychosis in Youths at Clinically High Risk. JAMA Psychiatry 75, 960-968, doi:10.1001/jamapsychiatry.2018.1543 (2018).

27. Aberizk, K. et al. Life Event Stress and Reduced Cortical Thickness in Youth at Clinical High Risk for Psychosis and Healthy Control Subjects. Biological Psychiatry: Cognitive Neuroscience and Neuroimaging, doi:https://doi.org/10.1016/j.bpsc.2021.04.011 (2021). 
28. Chaddad, A., Desrosiers, C. \& Toews, M. Multi-scale radiomic analysis of sub-cortical regions in MRI related to autism, gender and age. Scientific Reports 7, 45639, doi:10.1038/srep45639 (2017).

29. Davatzikos, C. et al. Whole-Brain Morphometric Study of Schizophrenia Revealing a Spatially Complex Set of Focal Abnormalities. Archives of General Psychiatry 62, 1218-1227, doi:10.1001/archpsyc.62.11.1218 (2005).

30. O'Connor, J. P. B. et al. Imaging biomarker roadmap for cancer studies. Nature Reviews Clinical Oncology 14, 169-186, doi:10.1038/nrclinonc.2016.162 (2017).

31. Li, T.-R. et al. Radiomics Analysis of Magnetic Resonance Imaging Facilitates the Identification of Preclinical Alzheimer's Disease: An Exploratory Study. Frontiers in Cell and Developmental Biology 8, doi:10.3389/fcell.2020.605734 (2020).

32. $\mathrm{Hu}, \mathrm{X}$. et al. Multivariate radiomics models based on 18F-FDG hybrid PET/MRI for distinguishing between Parkinson's disease and multiple system atrophy. European Journal of Nuclear Medicine and Molecular Imaging 48, 3469-3481, doi:10.1007/s00259-021-05325-z (2021).

33. Korda, A. I. et al. Identification of voxel-based texture abnormalities as new biomarkers for schizophrenia and major depressive patients using layer-wise relevance propagation on deep learning decisions. Psychiatry Research: Neuroimaging 313, 111303, doi:https://doi.org/10.1016/j.pscychresns.2021.111303 (2021).

34. Park, Y. W. et al. Differentiating patients with schizophrenia from healthy controls by hippocampal subfields using radiomics. Schizophrenia Research 223, 337-344, doi:https://doi.org/10.1016/j.schres.2020.09.009 (2020).

35. Bang, M. et al. An interpretable multiparametric radiomics model for the diagnosis of schizophrenia using magnetic resonance imaging of the corpus callosum. Translational Psychiatry 11, 462, doi:10.1038/s41398-021-01586-2 (2021).

36. Radulescu, E. et al. Grey-matter texture abnormalities and reduced hippocampal volume are distinguishing features of schizophrenia. Psychiatry Research: Neuroimaging 223, 179-186, doi:https://doi.org/10.1016/j.pscychresns.2014.05.014 (2014).

37. Hassan, I. et al. Radiomic Texture Analysis Mapping Predicts Areas of True Functional MRI Activity. Scientific Reports 6, 25295, doi:10.1038/srep25295 (2016).

38. Traverso, A., Wee, L., Dekker, A. \& Gillies, R. Repeatability and Reproducibility of Radiomic Features: A Systematic Review. International Journal of Radiation Oncology*Biology*Physics 102, 1143-1158, doi:https://doi.org/10.1016/j.ijrobp.2018.05.053 (2018).

39. Jha, A. K. et al. Repeatability and reproducibility study of radiomic features on a phantom and human cohort. Scientific Reports 11, 2055, doi:10.1038/s41598-021-81526-8 (2021).

40. Bach, S. et al. On Pixel-Wise Explanations for Non-Linear Classifier Decisions by Layer-Wise Relevance Propagation. PLOS ONE 10, e0130140, doi:10.1371/journal.pone.0130140 (2015).

41. Korda, A. I., Andreou, C. \& Borgwardt, S. Pattern classification as decision support tool in antipsychotic treatment algorithms. Experimental Neurology 339, 113635, doi:https://doi.org/10.1016/j.expneurol.2021.113635 (2021). 
42. Cui, L.-B. et al. Thalamus Radiomics-Based Disease Identification and Prediction of Early Treatment Response for Schizophrenia. Frontiers in Neuroscience 15, 764 (2021).

43. Masias, M. et al. S25. FRAMEWORK FOR THE CHARACTERIZATION OF HIPPOCAMPUS USING RADIOMIC APPROACH IN FIRST-EPISODE PSYCHOSIS. Schizophrenia Bulletin 46, S40-S40, doi:10.1093/schbul/sbaa031.091 (2020).

44. Riecher-Rössler, A. et al. The Basel early-detection-of-psychosis (FEPSY)-study - design and preliminary results. Acta Psychiatrica Scandinavica 115, 114-125, doi:https://doi.org/10.1111/j.1600-0447.2006.00854.x (2007).

45. Riecher-Rössler, A. et al. Das Basel Screening Instrument für Psychosen (BSIP): Entwicklung, Aufbau, Reliabilität und Validität. Fortschr Neurol Psychiatr 76, 207-216 (2008).

46. Yung, A. R. et al. Prediction of psychosis: A step towards indicated prevention of schizophrenia. British Journal of Psychiatry 172, 14-20, doi:10.1192/S0007125000297602 (1998).

47. Koutsouleris, N. et al. Use of Neuroanatomical Pattern Classification to Identify Subjects in At-Risk Mental States of Psychosis and Predict Disease Transition. Archives of General Psychiatry 66, 700712, doi:10.1001/archgenpsychiatry.2009.62 (2009).

48. Koutsouleris, N. et al. Detecting the Psychosis Prodrome Across High-Risk Populations Using Neuroanatomical Biomarkers. Schizophrenia Bulletin 41, 471-482, doi:10.1093/schbul/sbu078 (2015).

49. Coupe, P. et al. An Optimized Blockwise Nonlocal Means Denoising Filter for 3-D Magnetic Resonance Images. IEEE Transactions on Medical Imaging 27, 425-441, doi:10.1109/TMI.2007.906087 (2008).

50. Wiest-Daesslé, N., Prima, S., Coupé, P., Morrissey, S. P. \& Barillot, C. Rician noise removal by non-Local Means filtering for low signal-to-noise ratio MRI: applications to DT-MRI. Med Image Comput Comput Assist Interv 11, 171-179, doi:10.1007/978-3-540-85990-1_21 (2008).

51. Rajapakse, J. C., Giedd, J. N. \& Rapoport, J. L. Statistical approach to segmentation of single-channel cerebral MR images. IEEE Transactions on Medical Imaging 16, 176-186, doi:10.1109/42.563663 (1997).

52. Manjón, J. V. et al. Robust MRI brain tissue parameter estimation by multistage outlier rejection. Magnetic Resonance in Medicine 59, 866-873, doi:https://doi.org/10.1002/mrm.21521 (2008).

53. Cuadra, M. B., Cammoun, L., Butz, T., Cuisenaire, O. \& Thiran, J. Comparison and validation of tissue modelization and statistical classification methods in T1-weighted MR brain images. IEEE Transactions on Medical Imaging 24, 1548-1565, doi:10.1109/TMI.2005.857652 (2005).

54. Good, C. D. et al. A Voxel-Based Morphometric Study of Ageing in 465 Normal Adult Human Brains. Neurolmage 14, 21-36, doi:https://doi.org/10.1006/nimg.2001.0786 (2001).

55. van Timmeren, J. E., Cester, D., Tanadini-Lang, S., Alkadhi, H. \& Baessler, B. Radiomics in medical imaging-“how-to" guide and critical reflection. Insights into Imaging 11, 91, doi:10.1186/s13244020-00887-2 (2020). 
56. Tsai, F., Chang, C.-K., Rau, J.-Y., Lin, T.-H. \& Liu, G.-R. in Energy Minimization Methods in Computer Vision and Pattern Recognition. (eds Alan L. Yuille, Song-Chun Zhu, Daniel Cremers, \& Yongtian Wang) 429-440 (Springer Berlin Heidelberg).

57. Nardone, V. et al. Texture analysis as a predictor of radiation-induced xerostomia in head and neck patients undergoing IMRT. La radiologia medica 123, 415-423, doi:10.1007/s11547-017-0850-7 (2018).

58. Zhang, Y. et al. The Diagnostic Value of MRI-Based Texture Analysis in Discrimination of Tumors Located in Posterior Fossa: A Preliminary Study. Frontiers in Neuroscience 13, doi:10.3389/fnins.2019.01113 (2019).

59. Eichkitz, C. G., Amtmann, J. \& Schreilechner, M. G. Calculation of grey level co-occurrence matrixbased seismic attributes in three dimensions. Computers \& Geosciences 60, 176-183, doi:https://doi.org/10.1016/j.cageo.2013.07.006 (2013).

60. Brynolfsson, P. et al. Haralick texture features from apparent diffusion coefficient (ADC) MRI images depend on imaging and pre-processing parameters. Scientific Reports 7, 4041, doi:10.1038/s41598017-04151-4 (2017).

61. Zanetti, M. V. et al. Neuroanatomical pattern classification in a population-based sample of firstepisode schizophrenia. Progress in Neuro-Psychopharmacology and Biological Psychiatry 43, 116125, doi:https://doi.org/10.1016/j.pnpbp.2012.12.005 (2013).

62. Lourakis, M. A Brief Description of the Levenberg-Marquardt Algorithm Implemened by levmar. A Brief Description of the Levenberg-Marquardt Algorithm Implemented by Levmar 4 (2005).

63. Hua, K. et al. Tract probability maps in stereotaxic spaces: analyses of white matter anatomy and tract-specific quantification. Neurolmage 39, 336-347, doi:10.1016/j.neuroimage.2007.07.053 (2008).

64. Stelzer, J., Lacosse, E., Bause, J., Scheffler, K. \& Lohmann, G. Brainglance: Visualizing Group Level MRI Data at One Glance. Frontiers in Neuroscience 13, doi:10.3389/fnins.2019.00972 (2019).

65. Frey Brendan, J. \& Dueck, D. Clustering by Passing Messages Between Data Points. Science 315, 972-976, doi:10.1126/science.1136800 (2007).

66. Adriano, F., Spoletini, I., Caltagirone, C. \& Spalletta, G. Updated meta-analyses reveal thalamus volume reduction in patients with first-episode and chronic schizophrenia. Schizophrenia Research 123, 1-14, doi:https://doi.org/10.1016/j.schres.2010.07.007 (2010).

67. Haijma, S. V. et al. Brain volumes in schizophrenia: a meta-analysis in over 18000 subjects. Schizophrenia bulletin 39, 1129-1138, doi:10.1093/schbul/sbs118 (2013).

68. Huang, X. et al. Decreased Left Putamen and Thalamus Volume Correlates with Delusions in FirstEpisode Schizophrenia Patients. Frontiers in Psychiatry 8, 245 (2017).

69. Borgwardt, S. J. et al. Structural brain abnormalities in individuals with an at-risk mental state who later develop psychosis. British Journal of Psychiatry 191, s69-s75, doi:10.1192/bjp.191.51.s69 (2007). 
70. Pantelis, C. et al. Neuroanatomical abnormalities before and after onset of psychosis: a crosssectional and longitudinal MRI comparison. The Lancet 361, 281-288, doi:https://doi.org/10.1016/S0140-6736(03)12323-9 (2003).

71. Li, X.-B. et al. Altered resting-state functional connectivity of the insula in individuals with clinical high-risk and patients with first-episode schizophrenia. Psychiatry Research 282, 112608, doi:https://doi.org/10.1016/j.psychres.2019.112608 (2019).

72. Liang, S. et al. Classification of First-Episode Schizophrenia Using Multimodal Brain Features: A Combined Structural and Diffusion Imaging Study. Schizophrenia Bulletin 45, 591-599, doi:10.1093/schbul/sby091 (2018).

73. Pantelis, C. et al. Structural Brain Imaging Evidence for Multiple Pathological Processes at Different Stages of Brain Development in Schizophrenia. Schizophrenia Bulletin 31, 672-696, doi:10.1093/schbul/sbi034 (2005).

74. Torres, U. S. et al. Patterns of regional gray matter loss at different stages of schizophrenia: A multisite, cross-sectional VBM study in first-episode and chronic illness. Neurolmage: Clinical 12,115, doi:https://doi.org/10.1016/j.nicl.2016.06.002 (2016).

75. Zhao, C. et al. Structural and functional brain abnormalities in schizophrenia: A cross-sectional study at different stages of the disease. Progress in Neuro-Psychopharmacology and Biological Psychiatry 83, 27-32, doi:https://doi.org/10.1016/j.pnpbp.2017.12.017 (2018).

76. Sheffield, J. M. et al. Insula sub-regions across the psychosis spectrum: morphology and clinical correlates. Translational Psychiatry 11, 346, doi:10.1038/s41398-021-01461-0 (2021).

77. Ebdrup, B. H. et al. Hippocampal and caudate volume reductions in antipsychotic-naive first-episode schizophrenia. J Psychiatry Neurosci 35, 95-104, doi:10.1503/jpn.090049 (2010).

78. Crespo-Facorro, B. et al. Caudate nucleus volume and its clinical and cognitive correlations in first episode schizophrenia. Schizophrenia Research 91, 87-96, doi:https://doi.org/10.1016/j.schres.2006.12.015 (2007).

79. Fan, F. et al. Subcortical structures and cognitive dysfunction in first episode schizophrenia. Psychiatry Research: Neuroimaging 286, 69-75, doi:https://doi.org/10.1016/j.pscychresns.2019.01.003 (2019).

80. Curtis, M. T., Coffman, B. A. \& Salisbury, D. F. Parahippocampal area three gray matter is reduced in first-episode schizophrenia spectrum: Discovery and replication samples. Human Brain Mapping 42, 724-736, doi:https://doi.org/10.1002/hbm.25256 (2021).

81. Schultz, C. C. et al. Increased parahippocampal and lingual gyrification in first-episode schizophrenia. Schizophrenia Research 123, 137-144, doi:https://doi.org/10.1016/j.schres.2010.08.033 (2010).

82. Di Biase, M. A. et al. White matter connectivity disruptions in early and chronic schizophrenia. Psychological Medicine 47, 2797-2810, doi:10.1017/S0033291717001313 (2017).

83. Peters, B. D. \& Karlsgodt, K. H. White matter development in the early stages of psychosis. Schizophrenia Research 161, 61-69, doi:https://doi.org/10.1016/j.schres.2014.05.021 (2015). 
84. Walterfang, M. et al. White matter volume changes in people who develop psychosis. British Journal of Psychiatry 193, 210-215, doi:10.1192/bjp.bp.107.043463 (2008).

85. Surbeck, W. et al. Anatomical integrity within the inferior fronto-occipital fasciculus and semantic processing deficits in schizophrenia spectrum disorders. Schizophrenia Research 218, 267-275, doi:https://doi.org/10.1016/j.schres.2019.12.025 (2020).

86. Witthaus, H. et al. White matter abnormalities in subjects at ultra high-risk for schizophrenia and firstepisode schizophrenic patients. Schizophrenia Research 102, 141-149, doi:https://doi.org/10.1016/j.schres.2008.03.022 (2008).

87. Karlsgodt, K. H. et al. Diffusion Tensor Imaging of the Superior Longitudinal Fasciculus and Working Memory in Recent-Onset Schizophrenia. Biological Psychiatry 63, 512-518, doi:https://doi.org/10.1016/j.biopsych.2007.06.017 (2008).

88. Krakauer, K. et al. Patterns of white matter microstructure in individuals at ultra-high-risk for psychosis: associations to level of functioning and clinical symptoms. Psychological Medicine 47, 2689-2707, doi:10.1017/S0033291717001210 (2017).

89. Waszczuk, K. et al. Disturbances in White Matter Integrity in the Ultra-High-Risk Psychosis State-A Systematic Review. Journal of Clinical Medicine 10, doi:10.3390/jcm10112515 (2021).

90. Epstein, K. A. et al. White Matter Abnormalities and Cognitive Impairment in Early-Onset Schizophrenia-Spectrum Disorders. Journal of the American Academy of Child \& Adolescent Psychiatry 53, 362-372.e362, doi:https://doi.org/10.1016/j.jaac.2013.12.007 (2014).

91. Bloemen, O. J. N. et al. White-matter markers for psychosis in a prospective ultra-high-risk cohort. Psychological Medicine 40, 1297-1304, doi:10.1017/S0033291709991711 (2010).

92. Kubicki, M. \& Lyall, A. E. Antipsychotics and Their Impact on Cerebral White Matter: Part of the Problem or Part of the Solution? American Journal of Psychiatry 175, 1056-1057, doi:10.1176/appi.ajp.2018.18060752 (2018).

93. Szeszko, P. R. et al. White Matter Changes Associated with Antipsychotic Treatment in First-Episode Psychosis. Neuropsychopharmacology 39, 1324-1331, doi:10.1038/npp.2013.288 (2014).

94. Ho, B.-C., Andreasen, N. C., Ziebell, S., Pierson, R. \& Magnotta, V. Long-term Antipsychotic Treatment and Brain Volumes: A Longitudinal Study of First-Episode Schizophrenia. Archives of General Psychiatry 68, 128-137, doi:10.1001/archgenpsychiatry.2010.199 (2011).

95. Vita, A. \& De Peri, L. in Brain evolution, language and psychopathology in schizophrenia. Explorations in mental health. 212-228 (Routledge/Taylor \& Francis Group, 2014).

\section{Figures}



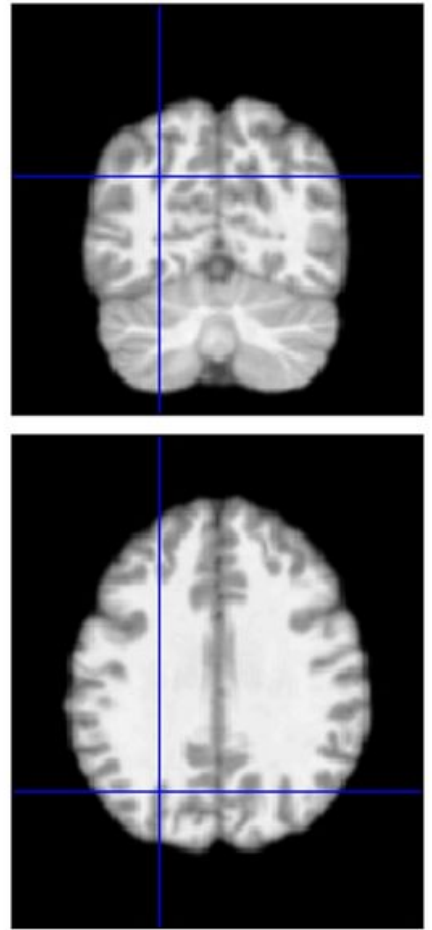

A

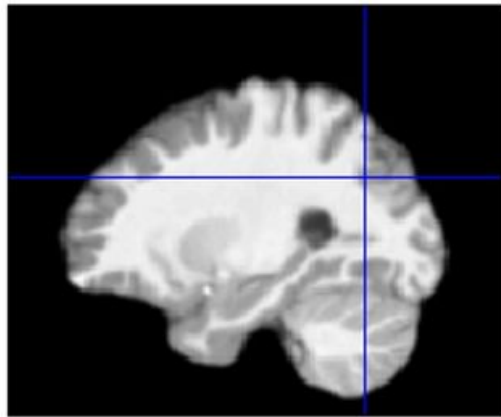

$Y=0.650323$
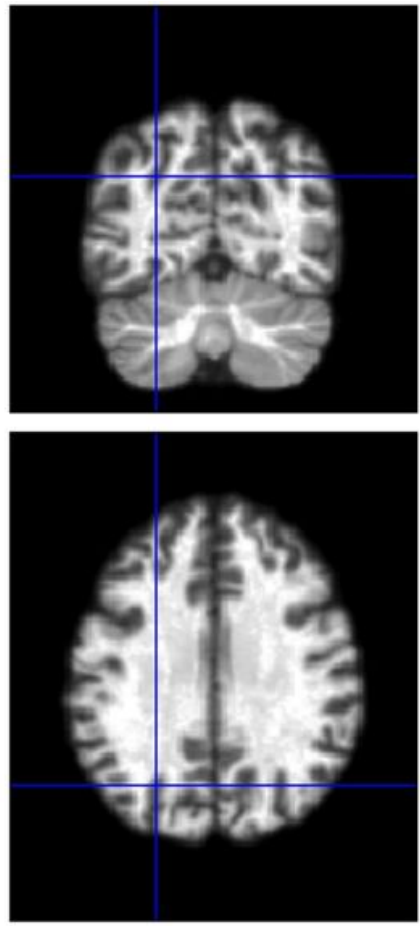

B

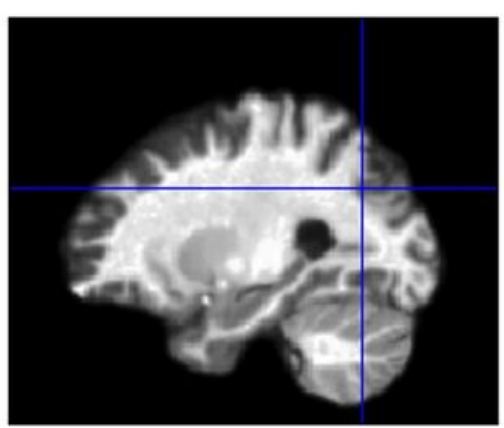

$\mathrm{Y}=0.83121$

Figure 1

Representation of the brain MRI in SPM12 for the a) initial MR image and b) transformed MR image using the histogram equalization.
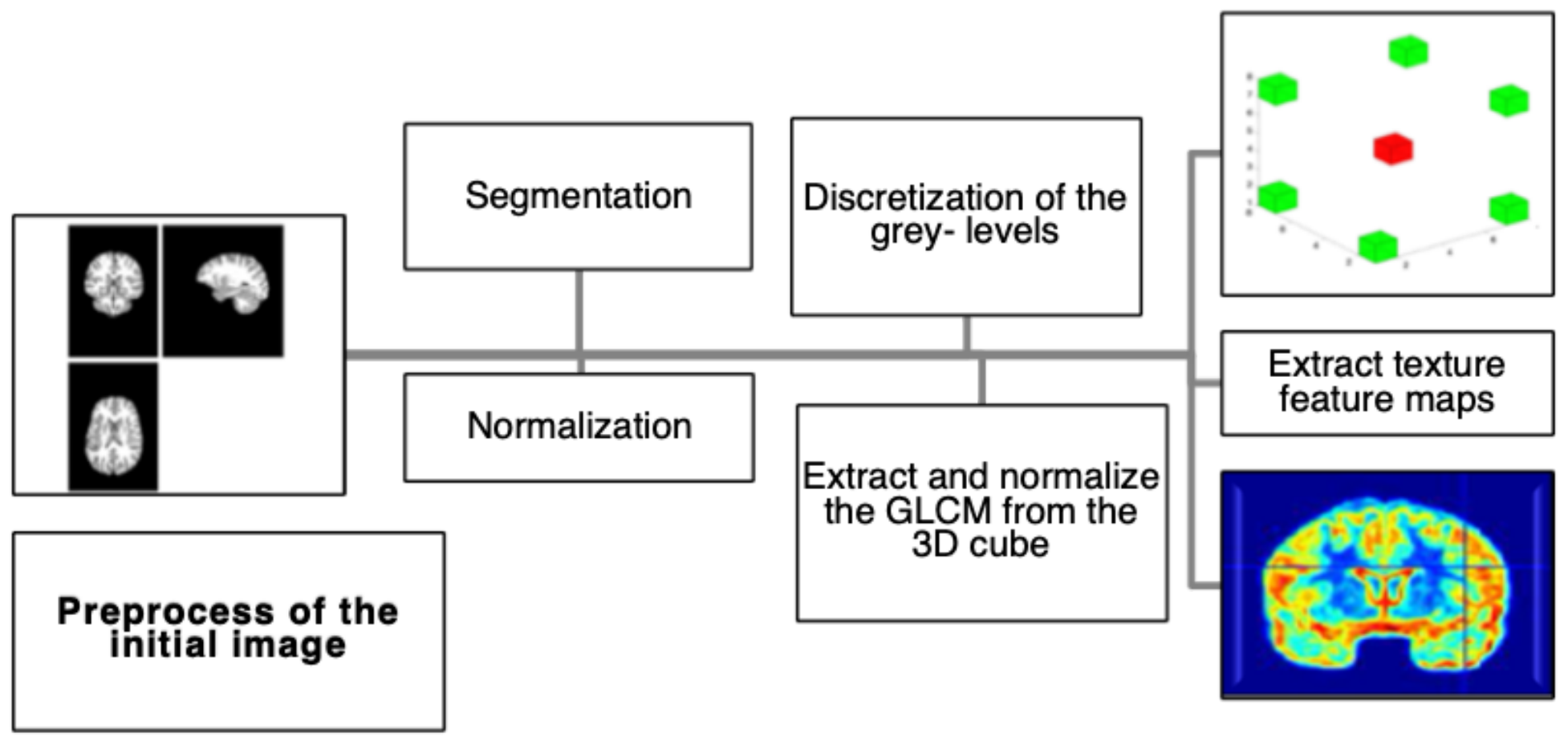

Figure 2 
Workflow for the calculation of the texture feature maps.

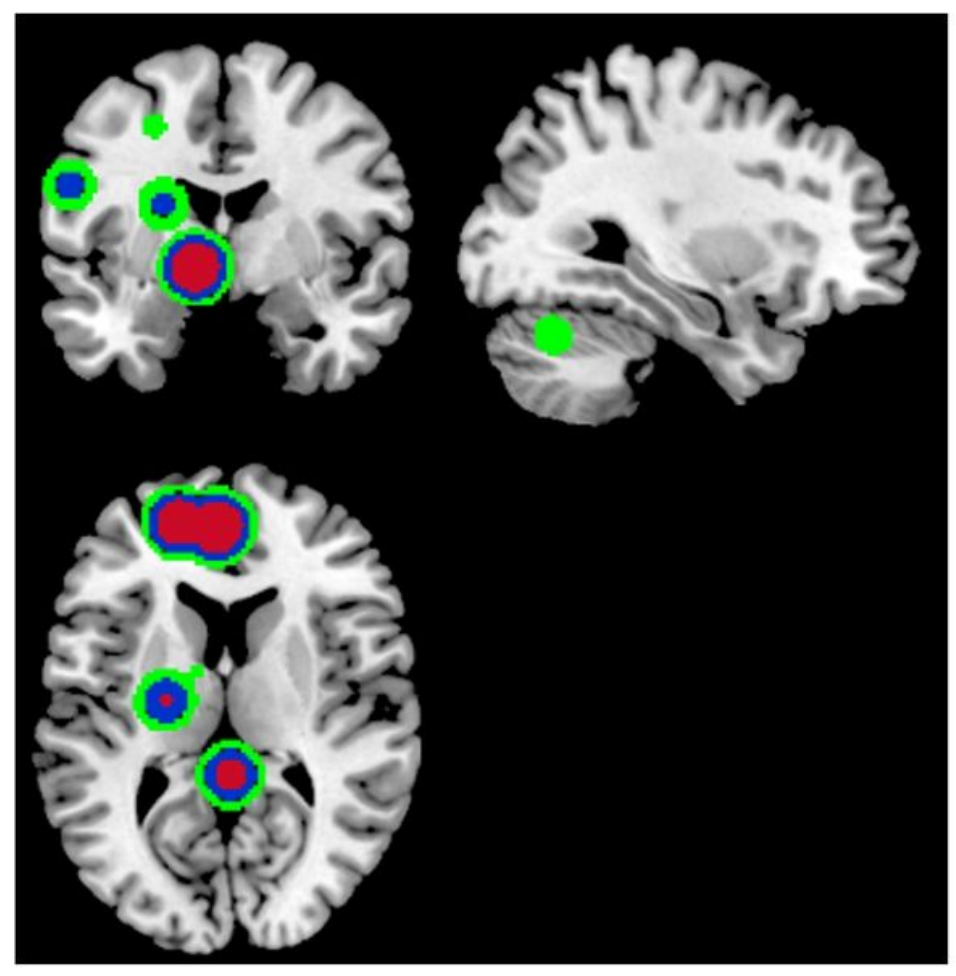

A

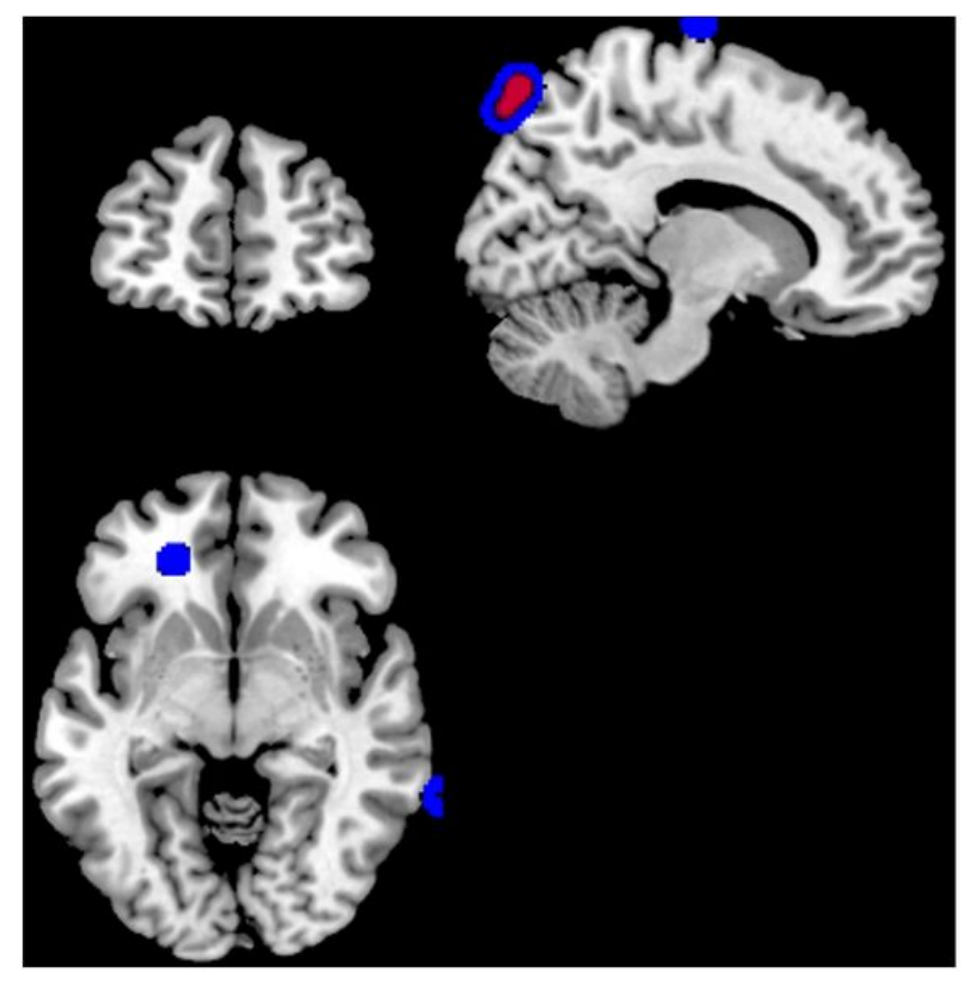

B

\section{Figure 3}

We demonstrated the PR of the correct classified subjects of each group against the other in classification schema a), FEP vs. HC for the registered texture feature map: a) difference of entropy and b) contrast. The red (cluster 1), blue (cluster 2 ) and green (cluster 3 ) color corresponds to the sorted clusters according to the number of subjects belong to each cluster. 


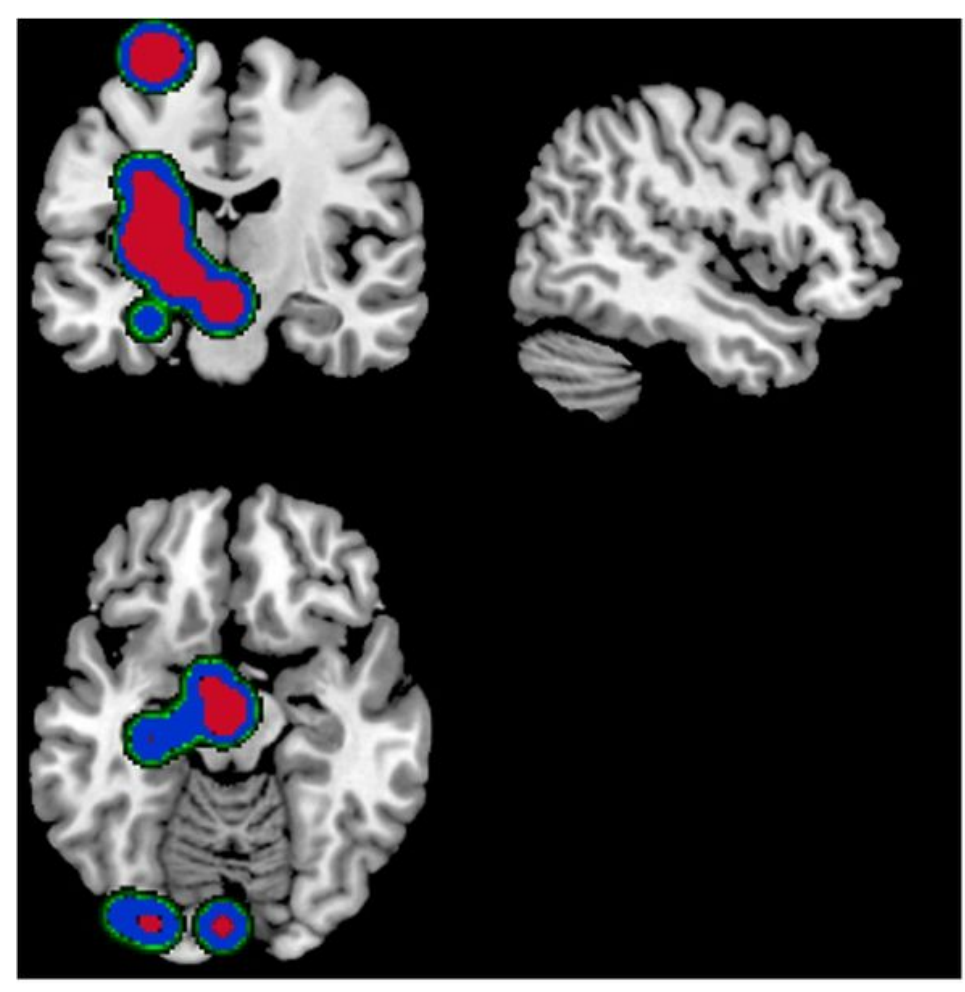

A

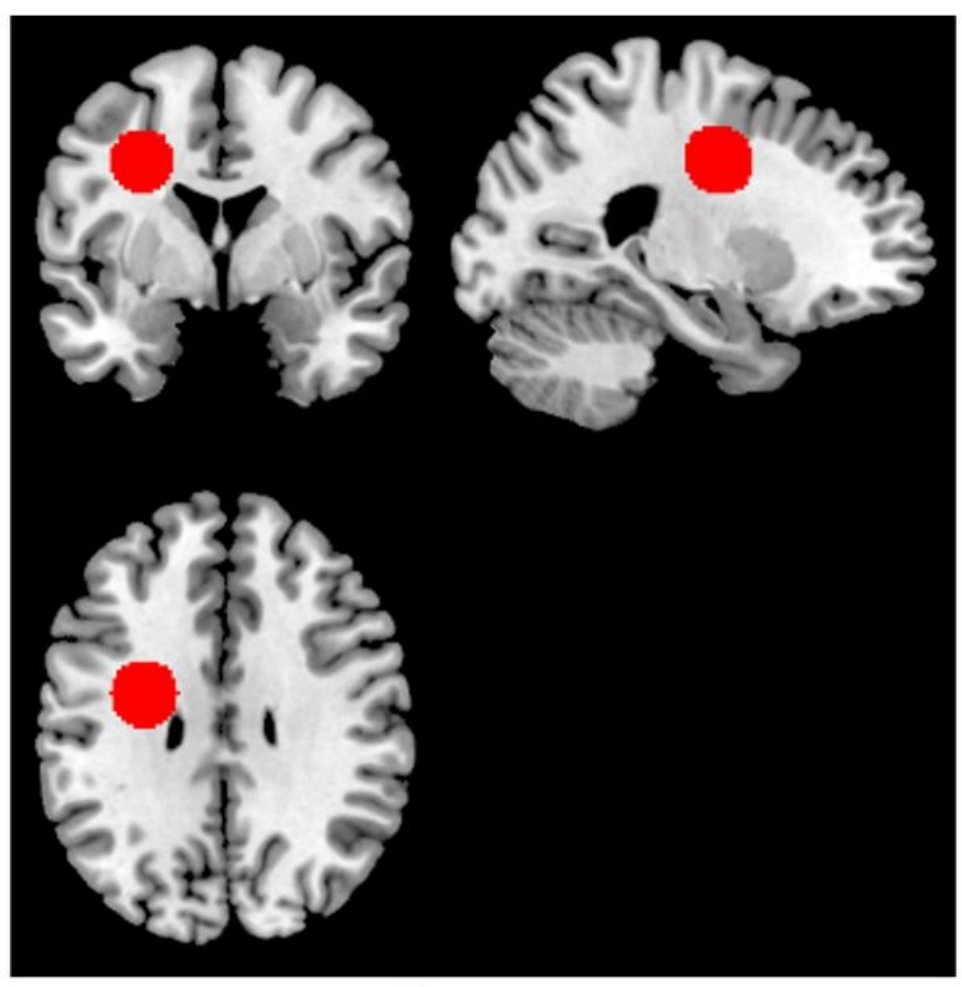

B

Figure 4

We demonstrated the PR of the correct classified subjects of each group against the other in classification schema b), FEP vs. CHR_NT for the registered texture feature map: a) difference of entropy and b) contrast. The red (cluster 1 ), blue (cluster 2) and green (cluster 3 ) color corresponds to the sorted clusters according to the number of subjects belong to each cluster. 


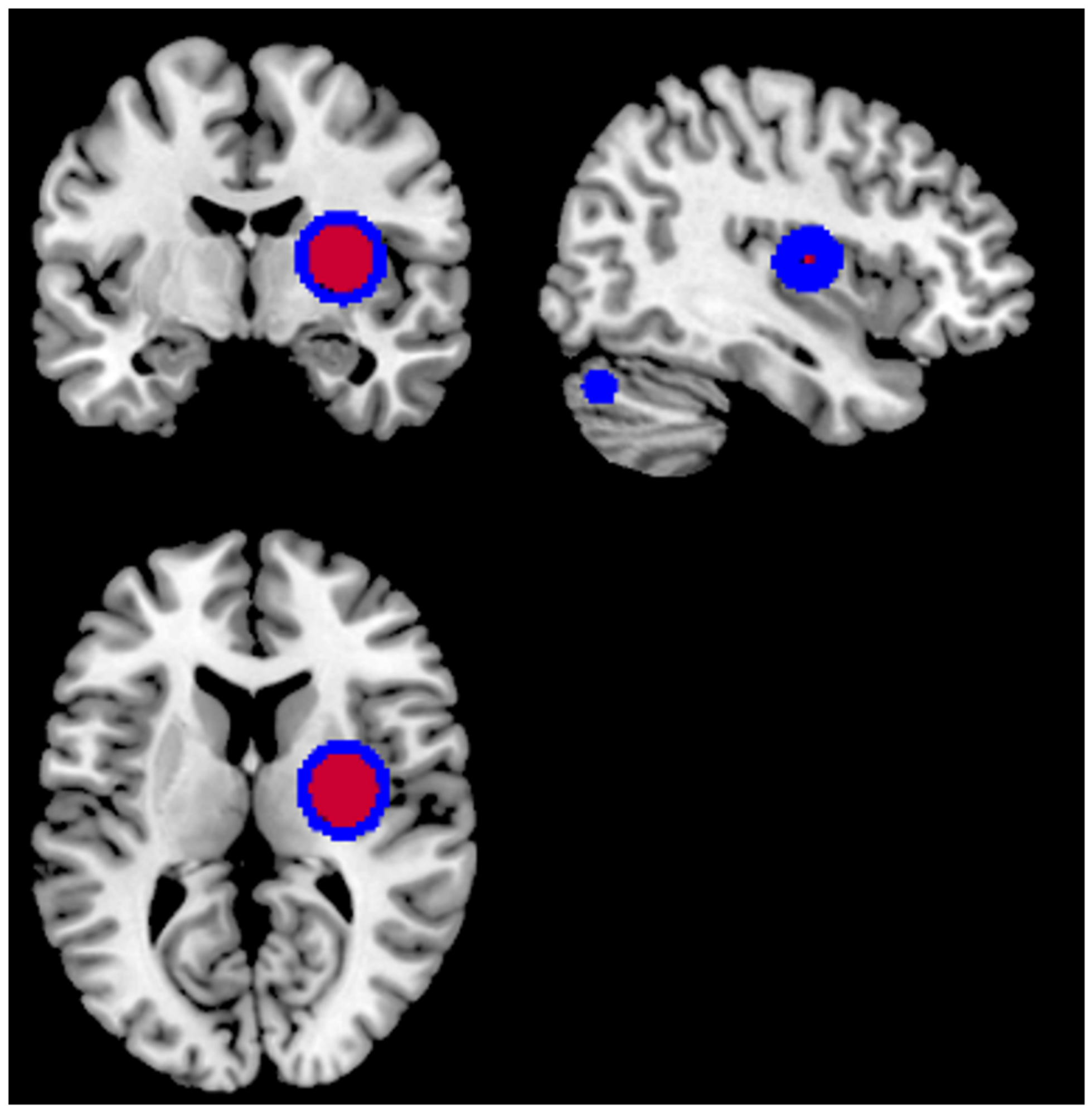

Figure 5

Classification of CHR_T as FEP using the difference of entropy in classification schema b). The red (cluster 1) and blue (cluster 2) color corresponds to the sorted clusters according to the number of subjects.

\section{Supplementary Files}


This is a list of supplementary files associated with this preprint. Click to download.

- Appendices.docx

- SupplementTABasel.docx 